\title{
基于红外阵列传感器的人体行为识别系统研究
}

\author{
王召军，许志猛，陈良琴 \\ (福州大学 物理与信息工程学院, 福建 福州 350108)
}

\begin{abstract}
摘要: 随着人口老龄化的到来，为了避免发生意外事故，对老人日常活动行为进行识别和监测的安全 监护系统的需求不断增长。传统的基于摄像头拍摄或者穿戴式传感器的活动状态监测系统存在着隐私 保护和使用不方便等不足。为此, 本文设计一种基于红外阵列传感器的人体行为识别系统。该系统通 过检测环境中的温度分布和变化情况识别人体行为, 不需要在老人身上佩戴任何设备, 尺寸小易于安 装，在黑暗环境中可正常工作，且由于采集到的是低分辨率信息，不会造成隐私泄露，对比传统方案 具有明显优势。从采集到的温度分布信息中提取特征并采用 $\mathrm{K}$ 最近邻（K-Nearest Neighbor, KNN）算 法实现了“走”、“坐”和 “跌倒” 3 种状态的识别。实验结果表明平均准确率可达到 95\%, 其中跌 倒准确率为 $97.5 \%$, 行走准确率高达 $100 \%$, 坐下准确率为 $92.5 \%$ 。
\end{abstract}

关键词: 行为识别; 红外阵列传感器; 多特征提取; K-近邻算法

中图分类号：TP391.4 文献标识码：A 文章编号：1001-8891(2020)05-0231-07

\section{Human Behavior Recognition System Based on Infrared Array Sensors}

\author{
WANG Zhaojun, XU Zhimeng, CHEN Liangqin \\ (College of Physics and Information Engineering, Fuzhou University, Fuzhou 350108, China)
}

\begin{abstract}
With the increase in the aging population, the demand to identify and monitor the daily activities of the elderly is growing. A monitoring system can effectively prevent accidents of elderly people. The traditional activity monitoring system based on the use of camera or wearable sensors has issues, such as privacy violations and inconvenience of use. Therefore, this study designs a human behavior recognition system based on infrared array sensors. The system recognizes activities on different temperature distributions in the environment. There is no need for the sensor to be worn by the elderly. The sensor is small in size, easy to install indoors, and can work in the dark. In addition, the data acquired by the sensor have a low resolution; therefore, there is no privacy violation. The designed system has significant advantages over the traditional systems. The features are extracted from the obtained temperature data, and the K-nearest neighbors algorithm is used to identify the three poses of "walking," "sitting," and "falling." The experimental results show that the average accuracy can reach 95\%, of which the accuracies for falling, walking, and sitting are $97.5 \%, 100 \%$, and $92.5 \%$, respectively.
\end{abstract}

Key words: activity recognition, infrared array sensor, multi-feature extraction, KNN algorithm

\section{0 引言}

随着科学和医疗技术快速发展, 人类的整体寿命 逐渐延长, 人口老龄化成了全球问题。随着老人的身 体机能慢慢减弱, 跌倒及其引起的伤害已成为老年人 健康和安全的重要威胁 ${ }^{[1]}$ 。在中国有 1.3 亿老年人, 每年出现超过 2500 万次跌倒事故 ${ }^{[2]}$ 。如何保障老人的
日常生活安全已成为家庭和社会必须面对的重大问 题。因此, 对于老人特别是独居老人的健康状态和行 动安全提供监测的系统得到了广泛关注。

现阶段, 针对跌倒检测国内外主要有 4 类技术 ${ }^{[3-6]}$ : 基于智能手机的跌倒检测、基于可穿戴式设备的跌倒 检测、基于视频的跌倒检测以及基于声学的跌倒检测 系统等。基于智能手机的检测方案，利用手机内置的

收稿日期：2019-05-09; 修订日期：2020-01-07

作者简介: 王召军 (1993-)，男，硕士研究生，研究方向：无线感知。E-mail:1678022616@qq.com。

通信作者: 许志猛 (1980-), 男, 副教授, 主要从事无线感知、无线通信与网络技术研究。E-mail:zhmxu@fzu.edu.cn。

基金项目: 国家自然科学基金资助项目（61401100); 福建省自然科学基金资助项目(2018J01805); 福州大学人才基金（GXRC-18083); 福州大学科研启 动基金（GXRC-18074）。 
加速度传感器、陀螺仪等可以实时采集使用者的状态 并通过算法判断是否跌倒, 由于该方案需要受监测对 象一直携带手机才能正常工作的限制, 使用上存在较 大弊端; 基于可穿戴式设备的方案与基于智能手机方 案缺点类似, 都要佩戴或者携带设备; 基于视频的检 测方案对使用者没有太多的限制, 但易受到光照影响 且容易造成隐私泄露, 在卧室、卫生间等场合不适合 使用; 基于声学的跌倒检测方案通过分析跌倒时对地 面的撞击, 提取声音或者地板震动等特征, 但由于其 他外物造成声音干扰的情况时常发生, 准确率较低。 为此, 日本的 Jihoon Hong 等人提出了使用松下 Grid-eye 的 $8 \times 8$ 红外阵列传感器设计一个跌倒检测 系统 ${ }^{[7]}$, 通过监测人体红外辐射温度分布情况识别跌 倒动作。2017 年, 上海大学的杨任兵等人提出了基于 同一款传感器采集的红外图像的多特征提取的跌倒 检测算法 ${ }^{[8]}$ 。传统视频监控一方面会暴露隐私, 另一 方面容易受到光照条件的影响造成在黑暗环境中无 法正常工作, 尤其在易发生滑倒的浴室、卫生间等环 境中会被雾气影响采集图像困难无法达到监测目的。 与视频监测相比, 红外方案对物体红外辐射温度进行 检测能够解决隐私保护、光照变化、阴影和夜间可视 性等问题, 应用场景更多; 视频图像采用模糊化处理 等技术避免隐私泄露, 仍然存在硬件被破解的问题, 而红外热电堆采集的是温度点阵, 硬件上无法获得清 晰图像, 隐私保护更安全可靠。考虑到对老人日常活 动行为的监测具有防范发生意外的重要意义, 虽然上 述两个基于红外阵列传感器的检测系统对于意外摔 倒有较好的监测效果, 但尚未实现对人体坐下、行走 等其他动作的识别。

为了解决以上问题, 本文基于德国海曼 (HEIMANN Sensor) 公司的 HTPA $32 \times 32$ 红外阵列 传感器设计了一个人体行为识别系统。

\section{1 行为识别系统设计}

\section{1 红外阵列传感器介绍}

红外传感器如图 1(a)所示, 其主要参数如表 1 所 示。该传感器内含热电堆元件, 可采集被探测物体表 面红外辐射, 不受光照因素影响, 在黑暗状况下也可 以探测人体的温度分布图像。由于该传感器分辨率 低, 即使将温度值转换成伪色彩图像也可以确保隐私 不被泄露, 如图 1(b)所示为一个手掌的红外温度分布 图, 从图中仅能看清目标轮廓, 细节信息不会泄露。

\section{2 系统构成}

人体活动识别系统整体结构如图 2 所示。系统主 要由红外阵列传感器、基于树莓派的温度数据采集平
台和基于个人计算机（Personal Computer, PC) 的动 作识别平台构成。红外阵列传感器吊装在天花板上, 由树莓派通过 $\mathrm{I}^{2} \mathrm{C}$ 接口实现控制, 实现探测区域的温 度分布信息的采集; 采集到的温度数据通过树莓派内 置的 WiFi 发送到 PC 端, PC 端的识别程序从温度分 布数据中提取 5 种特征, 分别经过 $K$ 最近邻算法 ${ }^{[9-10]}$ 、 支持向量机 (Support Vector Machine, SVM) 算法 ${ }^{[11-12]}$ 和卷积神经网络 (Convolutional Neural Network, CNN）算法 ${ }^{[13-16]}$ 进行分类, 并与数据库数据对比, 判 断温度分布数据对应人体的动作类别, 进而完成人体 动作的识别。

表 1 传感器的规格参数 Table1 Specifications of the sensor

\begin{tabular}{ll}
\hline Project & Model / Performance \\
\hline Model & HTPA $32 \times 32$ dR1L 5.0/0.85F7.7eHiC \\
\hline Number of pixels & $1024(32 \times 32)$ \\
\hline $\begin{array}{l}\text { Temperature range of } \\
\text { measurement object }\end{array}$ & $-40-85^{\circ} \mathrm{C}$ \\
\hline Angle & $66^{\circ}$ \\
\hline Frame rate & 60 frames per second or 2 frames per \\
& second \\
\hline $\begin{array}{l}\text { Temperature output } \\
\text { mode }\end{array}$ & $\mathrm{I}^{2} \mathrm{C}$ \\
\hline $\begin{array}{l}\text { Package size } \\
\text { Sensitivity }\end{array}$ & $\begin{array}{l}\text { Approx. } 450 \mathrm{~V} / \mathrm{W} \text { without optics and } \\
\text { filters }\end{array}$ \\
\hline $\begin{array}{l}\text { Thermal pixel time } \\
\text { constant }\end{array}$ & $<4$ ms \\
\hline
\end{tabular}

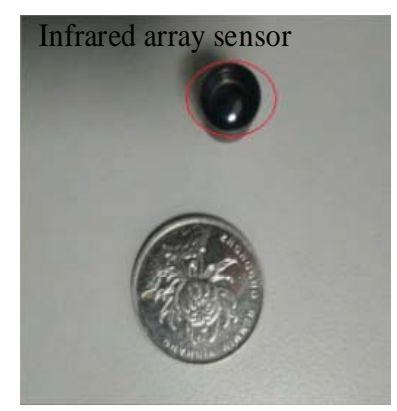

(a) 红外阵列传感器实物图

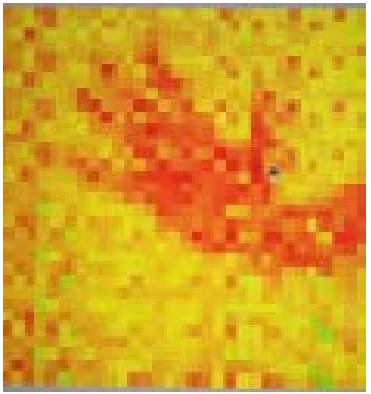

(b) 红外温度分布图 (a) Infrared array sensor physical map (b) Infrared temperature profile

图 1 红外阵列传感器 Fig.1 Infrared array sensor

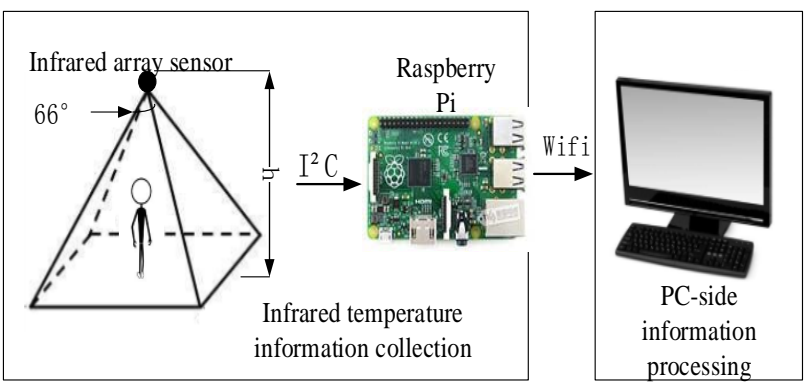

图 2 系统构成设计 Fig.2 System composition design 


\section{2 行为识别系统算法}

动作识别算法流程图如图 3 所示, 该算法分为 3 步: 人体检测, 提取特征和分类。PC 端接收到温度 分布数据后, 计算前后两帧或者两帧以上的连续最大 温度分布方差: 若前后连续两帧或者两帧以上的方差 均小于温度方差阈值, 则视为此时无人; 若前后连续 两帧或者两帧以上的方差均大于设定温度方差阈值, 则此时探测区域有活动的人存在。若存在活动的人, 提取数据特征与数据库中的训练数据对比分类, 进而 判断出发生的动作。

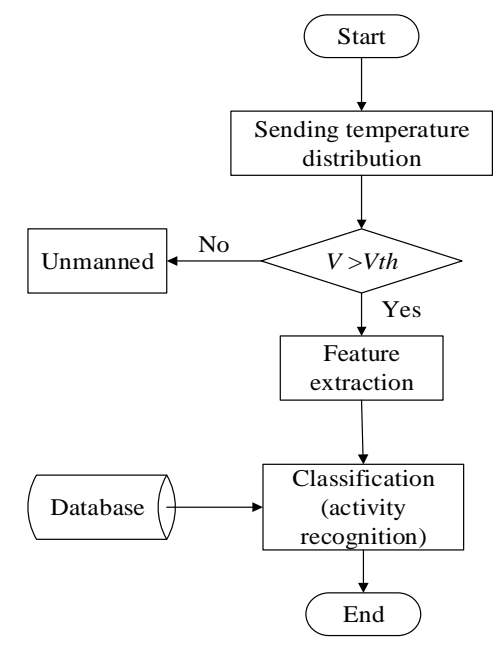

图 3 行为识别算法流程图

Fig.3 Flowchart of behavior recognition algorithm

\section{1 人体检测}

在室内无人活动场景下, 环境温度分布数据均匀 且变化小, 但有人存在且发生动作时, 温度分布数据 就会发生波动, 进而提取特征。

海曼 HTPA $32 \times 32$ 红外传感器发出的温度分布 第 $k$ 帧可以表示为:

$$
T_{k}=\left[\begin{array}{cccc}
a_{11 k} & a_{12 k} & \cdots & a_{132 k} \\
a_{21 k} & a_{22 k} & \cdots & a_{232 k} \\
\vdots & \vdots & & \vdots \\
a_{321 k} & a_{322 k} & \cdots & a_{3232 k}
\end{array}\right]
$$

式中: $a_{i j k}$ 是第 $k$ 帧的第 $(i, j)$ 个温度值。通过计算时间 窗宽度 $\tau$ 为 10 帧内的每个像素的方差判断是否有人。

当前帧的第 $(i, j)$ 像素的温度方差计算公式为:

$$
v_{i j k_{\mathrm{c}}}=\frac{1}{\tau} \sum_{k=k_{\mathrm{c}}-(\tau-1)}^{k_{\mathrm{c}}}\left(a_{i j k}-\overline{a_{i j k_{\mathrm{c}}}}\right)^{2}, \quad k_{\mathrm{c}} \geq \tau
$$

式中: $k_{\mathrm{c}}$ 为当前帧帧号。每获得一幅红外温度分布图 像都可以求出 1024 个单像素点的方差。探测区域有 活动的人存在时的温度方差比无人时大。 $\overline{a_{i j k_{c}}}$ 为连续 $\tau$
帧中当前帧 $(i, j)$ 像素点的温度平均值, 计算公式如下:

$$
\overline{a_{i j k_{\mathrm{c}}}}=\frac{1}{\tau} \sum_{k=k_{\mathrm{c}}-(\tau-1)}^{k_{\mathrm{c}}} a_{i j k}, \quad k_{\mathrm{c}} \geq \tau
$$

当前后连续两帧或两帧以上的温度分布方差大 于温度方差阈值 $V_{\mathrm{th}}$ 时, 则认为探测区域有活动的人 存在并提取特征。

温度方差阈值 $V_{\mathrm{th}}$ 的选择会影响算法的准确率, 选取太大, 漏检率升高, 选取太小, 增加计算量, 误 判率也会提高。因此, 本文阈值 $V_{\mathrm{th}}$ 的确定方法为选 择室内环境无人的情况下, 运行实验测试装置 $5 \mathrm{~min}$, 计算这段时长内的最大温度分布方差 $V$, 重复测试 5 次, 选择 5 次实验中的最大值作为阈值 $V_{\text {th }}$ 。通过实验 测试阈值选取为 2 。本实验通过计算温度方差来判断 检测区域是否存在活动的人。温度方差是由每帧内像 素点的温度值与滑窗内像素点平均值差值的平方和 决定, 因此当室内温度升高或者降低时, 每帧中像素 点温度值会增加或者降低, 滑窗内像素点平均值也随 之升高或者降低, 两者的差值变化较小, 温度方差阈 值受温度值影响较小。

\section{2 特征提取}

探测区域有活动的人存在时, 提取出 5 种关于人 体姿态的特征。当最大温度分布方差大于温差阈值 时, 提取平均有效像素点数量 $n$ 、激活帧 $K_{\mathrm{act}}$ 、反应 像素的最大数量 $P_{\max }$ 、最大温度分布方差 $V_{\max }$ 、有效 动作面积 $S_{\text {real }}$ 等 5 种特征, 图 4 中选取部分像素点方 差图像, 具体方法如下:

1) 平均有效像素点数量 $n$ : 根据红外热源的等效 模型 ${ }^{[17]}$ 可知, 人体可近似等效于直立的圆柱体, 高度 影响红外辐射强度, 且人体的上半躯干部分比下半部 分红外辐射能力强。红外传感器对人体敏感程度还和 运动方向有关, 红外传感器对于径向移动反应不敏 感, 对于横切方向移动最敏感。在室温阈值为 $15^{\circ} \mathrm{C}$ 的 环境下, 人体处于静态时, 测量出超过室温阈值的温 度像素点的总数量 $N$ 除以总帧数 $L$, 就是超过室温阈 值的单帧平均像素点数量, 即为平均有效像素点数量 $n$, 该特征可表示为:

$$
n=\frac{N}{L}
$$

静态下, 发生行走、坐下和跌倒时, 受高度影响, 行走时的单帧内平均有效像素点数量最多, 跌倒时的 单帧内平均有效像素点数量最少。

2 ) 激活帧 $K_{\mathrm{act}}$ : 当前后连续两帧或两帧以上的最 大温度分布方差大于温差阈值时, 则该帧为人体移动 的开始帧, 记为第 $k_{1}$ 帧; 当前后连续两帧或两帧以上 
最大温度分布方差小于温差阈值时, 则该帧为人体移 动的结束帧，记为第 $k_{2}$ 帧。如图 4 所示，该特征可表 示为:

$$
K_{\mathrm{act}}=k_{1}-k_{2}+1
$$

该特征表示发生动作所持续的时间, 若室内环境 中没有人，该特征值很小且基本不发生变化; 但当有 人存在并发生运动时, 该特征值会变大。在发生行走、 坐下、跌倒动作时, 行走所花费时间最长, 因此行走 的激活帧的值最大。

3) 反应像素的最大数量 $P_{\text {max }}$ : 表示在第 $k_{1}$ 帧到 第 $k_{2}$ 帧之间, 每帧的 1024 个单像素点数的方差大于 温差阈值时的最大像素数量。该特征表示红外传感器 下发生相应动作采集到的像素点的数量。在发生行 走、坐下、跌倒动作时, 由于高度因素, 行走时的反 应像素的数量最大, 如图 4 所示。

4）最大温度分布方差 $V_{\text {max }}$ ：表示在第 $k_{1}$ 帧到第 $k_{2}$ 帧之间的最大温度分布方差。该特征表示运动趋势 的变化程度。在发生行走、坐下、跌倒动作时, 跌倒 属于意外发生, 运动状态从站立状态急剧变化成平躺 状态, 运动趋势变化最大; 其次, 坐下的动作变化趋 势要比行走大, 因此跌倒的温度分布方差最大, 如图 4 所示。

5) 有效动作面积 $S_{\text {real }}$ 把人体发生动作近似轮廓 化成规则矩形, 计算发生动作近似面积大小即为有效 动作面积。通过测得超过激活帧中每一帧内的活动像 素点数量 $M$ 除以每平方的像素点数量 $m$ 即可得到该 帧上采集到的实际动作发生的大致面积, 该特征可表 示为:

$$
S_{\text {real }}=M / m
$$

有效动作面积 $S_{\text {real }}$ 不仅可以作为训练特征, 同时 也可以作为校验数据验证算法判断是否准确, 通过有 效动作面积与实际估算动作面积相比较, 即可判断出 发生哪种动作。

\section{3 分类算法}

通过与 SVM 算法和 CNN 算法的分类效果对比, 本文选择融合多特征且识别率高的 KNN 算法进行分 类。在分类中, 将测试数据与数据库中的训练数据进 行比较, 进而判断出发生的实际动作。KNN 算法原 理是如果一个样本在特征空间中距离 $k$ 个最相邻的样 本中的大多数属于某一个类别, 则该样本也属于这个 类别, 并具有这个类别上样本的特性, 一般距离用欧 氏距离度量。 $\mathrm{K}$ 值的选择会影响分类的准确性, $\mathrm{K}$ 值 太大增加算法计算时间, $\mathrm{K}$ 值太小容易过拟合, 因此, 使用交叉验证法 ${ }^{[18]}$ 确定 K 值。

\section{3 传感器采集数据的校准}

由公式(6)可知, 动作识别所采用的特征涉及人体 肢体在传感器检测数据中的面积计算。本系统采用的 实验模型及像素位置如图 5 所示, 从图中可以看出, 传感器的安装高度和角度会影响面积计算结果。为 此, 有必要根据传感器的安装高度和角度对采集到的 数据进行校准。

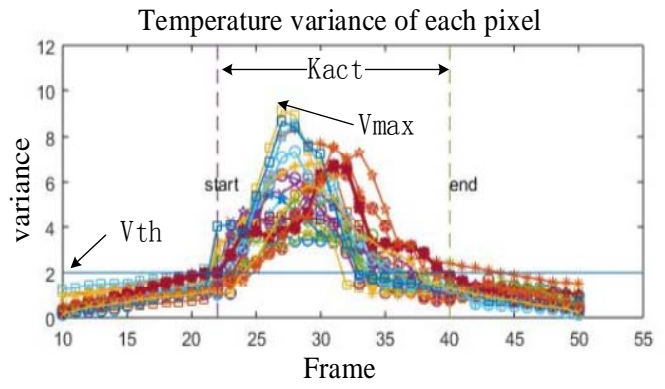

(a) 像素的温度方差 (a) Pixel temperature variance

Number of pixels exceeding the variance threshold

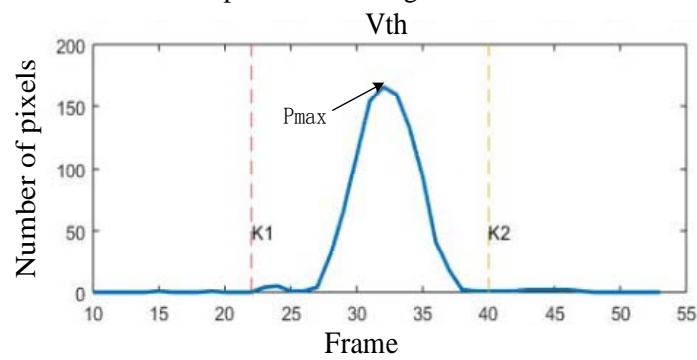

(b) 超过方差阈值的像素数量

(b) Number of pixels above the variance threshold

图 4 提取的特征: $K_{\mathrm{act}}, P_{\text {max }}$ 和 $V_{\text {max }}$

Fig.4 Extracted features: $K_{\mathrm{act}}, P_{\max }$ and $V_{\max }$

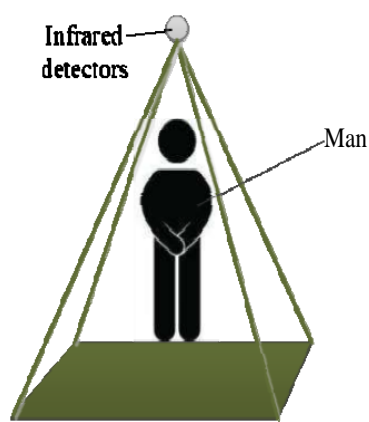

(a) 实验模型图

(a) Experimental model diagram

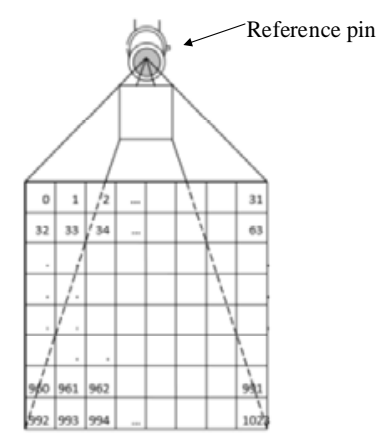

(b)像素位置图

(b) Pixel location map
图 5 实验模型及像素位置

Fig.5 Experimental model and pixel location

\section{1 根据安装角度的数据校准}

已知红外传感器视野角度为 $33^{\circ} \times 33^{\circ}$, 假设红外 传感器方向与垂直地面方向的夹角为 $\alpha$, 且 $0^{\circ}<\alpha<$ $57^{\circ}$, 传感器安装存在夹角的情况下, 特征值中使用 
到的探测面积有地面探测面积 $S_{\mathrm{d}}$ 和头部高度探测面 积 $S_{\text {head }}$ 两种。计算公式如下:

$$
S_{\mathrm{d}}=2 h^{2} \tan 33^{\circ}\left[\tan \left(\alpha+33^{\circ}\right)-\tan \left(\alpha-33^{\circ}\right)\right]
$$

式中: $h$ 表示红外传感器距离地面的垂直高度。

根据模型角度得出移动的人体头部高度模型探 测面积的计算公式如下:

$S_{\text {head }}=2(h-H)^{2} \tan 33^{\circ}\left[\tan \left(\alpha+33^{\circ}\right)-\tan \left(\alpha-33^{\circ}\right)\right]$

式中: $H$ 表示人的身高。

通过公式(6)计算有效动作面积 $S_{\text {real }}$, 可以得出:

$$
S_{\text {real }}=\frac{M}{m}=\frac{M \cdot S}{1024}
$$

式中: 根据探测的是头部还是地面的面积, $S$ 分别为 $S_{\text {head }}$ 和 $S_{\mathrm{d}}$ 。

将人体发生对应动作占地面积矩形化可知跌倒 估算面积大约为 $0.9 \sim 1.14 \mathrm{~m}^{2}$, 实际坐下估算面积大 约为 $0.25 \sim 0.48 \mathrm{~m}^{2}$, 实际行走估算面积大约为 $0.04 \sim$ $0.1 \mathrm{~m}^{2}$ 。通过将公式(7)、(8)代入公式(9)中, 可以计算 出发生动作的有效动作面积, 将有效动作面积和实际 估算面积对比, 即可判断出发生哪种动作。

\section{2 根据安装高度的数据校准}

在固定红外传感器高度时受到房间高度因素的 影响, 一般不超过 $3 \mathrm{~m}$, 结合一般人体身高因素, 选 择 $2 \mathrm{~m} 、 2.5 \mathrm{~m}$ 和 $3 \mathrm{~m}$ 试验校正, 人体身高选择 $1.8 \mathrm{~m}$ （人体身高的选择按照一般情况计算）。

当红外传感器方向与垂直地面方向的夹角为 $0^{\circ}$, 红外传感器固定高度分别为 $3 \mathrm{~m} 、 2.5 \mathrm{~m}$ 和 $2 \mathrm{~m}$ 时: 通 过公式(7)和(8)计算可知探测的地面最大面积分别约 为 $15.2 \mathrm{~m}^{2} 、 10.5 \mathrm{~m}^{2}$ 和 $6.7 \mathrm{~m}^{2}$, 因此每平方米的像素点 数量分别约为 68、97 和 151 个。同理可计算出人体 头顶高度平面上可探测的最大面积分别约为 $2.4 \mathrm{~m}^{2}$ 、 $0.83 \mathrm{~m}^{2}$ 和 $0.0675 \mathrm{~m}^{2}$, 计算出此时的每平方米的像素 点数量大约为 $420 、 1238$ 和 15175 个。通过测得在温 度方差超过方差阈值 $V_{\mathrm{th}}$ 的 $\left[k_{1}, k_{2}\right]$ 区间内像素点除以 每平方的像素点数量即可得到该实际动作有效动作 面积。由实验测得在红外传感器高度分别为 $3 \mathrm{~m} 、 2.5$ $\mathrm{m}$ 和 $2 \mathrm{~m}$ 时人行走时的像素点数量大约 $320 \sim 400$ 、 400 450 和 450 500 个, 跌倒时的像素点数量大约 120 130、130 150 和 150 200 个, 因此可计算出 实际行走时有效动作面积大小分别约为 $0.76 \sim 0.95$ $\mathrm{m}^{2} 、 0.32 \sim 0.36 \mathrm{~m}^{2}$ 和 $0.029 \sim 0.033 \mathrm{~m}^{2}$, 实际跌倒时的 有效动作面积大小分别约为 $1.76 \sim 2.94 \mathrm{~m}^{2} 、 1.3 \sim 1.5$ $\mathrm{m}^{2}$ 和 $0.99 \sim 1.32 \mathrm{~m}^{2}$ 。

综上所述, 当红外传感器高度为 $2 \mathrm{~m}$ 时有效动作 面积与实际估算面积最为符合, 因此红外传感器安装 高度为 $2 \mathrm{~m}$ 。
红外传感器经过安装角度及高度的校准, 将人体 发生有效动作面积与实际估算面积对比, 即可判断出 发生哪种动作。

\section{4 实验}

\section{1 实验装置}

实验使用德国海曼公司 HTPA32 $\times 32$ 红外阵列传 感器; 树莓派使用 3B 型号, 其系统基于 Linux, 有 SD/MicroSD 卡、内存、硬盘, 主板上有 USB 接口和 以太网接口, HDMI 高清视频输出接口、GPIO 接口, WiFi 通信模块, 实验装置如图 6(a)所示。

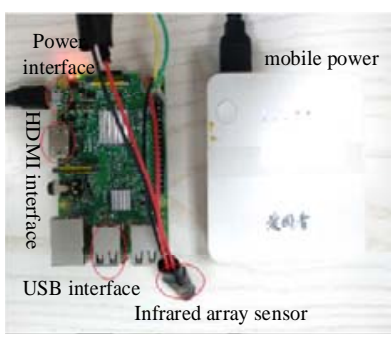

(a) 实验装置实物图

(a) Experimental device physical map (b) Simulated walking

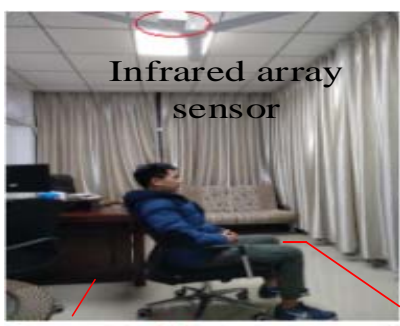

(c) 模拟坐下

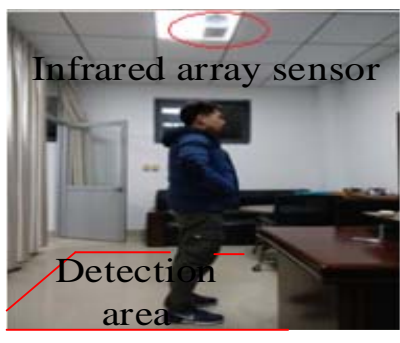

(b) 模拟行走

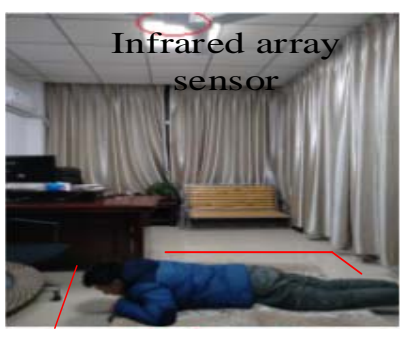

(d) 模拟跌倒 (c) Simulate sitting down

(d) Simulated fall

\section{2 实验环境}

本实验的实验环境选择室内普通办公房间, 室温 $15^{\circ} \mathrm{C}$ 左右, 实验测试场景如图 6(b)、(c)、(d) 所示。实 验装置为一个传感器垂直吊装在天花板上, 高度为 2 $\mathrm{m}$ 。被测实验人员需要根据要求完成行走、坐下和跌 倒 3 种动作。

\section{3 获取测试数据}

本文使用 KNN 算法和 SVM 算法实验时, 测试 8 名人员 (4 男 4 女), 要求被测人员在检测区域依次 完成行走、坐下和跌倒 3 种动作, 每种动作重复 10 次, 然后保存每次实验数据, 最后获得 240 组数据, 其中 SVM 算法进行分类时, 训练集为 122 组, 测试 集为 118 组, SVM 模型含有两个重要的参数 $c$ 和 $g$, 其中 $c$ 为惩罚参数取值范围为 $2^{-2} \sim 2^{4}, g$ 为选择径向 基函数（Radial Basis Function, RBF）后自带的参数, 取值范围为 $2^{-4} \sim 2^{4}$, 调整 $c$ 和 $g$, 当准确率最高时参 
数 $c$ 为 5 , 参数 $g$ 为 1.8 。使用 Keras 和 CNN 构建分 类器, 搭建的 VGGNet 结构包括 5 层卷积层、3 层池 化层和 1 层全连接层, 其中卷积层使用线性整流函数 (Rectified Linear Unit, ReLU) 为激活函数, 全连接层 使用 Softmax 分类器, 训练轮数与每次训练批次数量 的乘积要大于图片数量, 所以网络训练总次数设定为 500 轮, 每个训练批次数量为 32 , 依次改变学习率的 大小 $0.1 、 0.05 、 0.01 、 0.005 、 0.001 、 0.0005$ 以及 0.0001 , 统计行为动作识别准确率, 最后选择识别率最高时的 学习率 0.001 。使用 CNN 算法识别姿体动作实验时, 测试 6 名人员 (4 男 2 女), 总保存 11420 张图片, 其 中训练集 11120 张, 测试集 300 张, 实验行为动作模 拟结果如图 7 所示。



(a) 跌倒模拟图

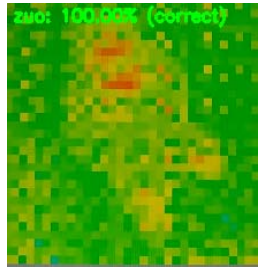

(b) 坐下模拟图 (a) Falling simulation (b) Sitting simulation

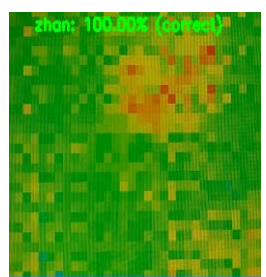

(c) 行走模拟图 $\quad$ (c) Walking simulation

图 7 姿态动作模拟图

Fig.7 Attitude motion simulation diagram

\subsection{KNN 算法最优 $K$ 值的选择}

本文使用交叉验证法确定最优 $K$ 值,一般 $K$ 值大 小不超过 10 。将实验测得的 240 组数据集, 按照不同 比例分成训练集和测试集, 以 3 为初始值, 2 为步长 依次选取不同 $K$ 值进行分类计算相对应的准确率, 选 择准确率最高的作为最优 $K$ 值。如表格 2 所示, 可得 出最优 $K$ 值为 3 。

\begin{tabular}{lllll} 
表 2 & 交叉验证结果 & Table 2 & \multicolumn{3}{c}{ Cross-validation results } \\
\hline \multicolumn{1}{l}{ Test set: training } & $K=3$ & $K=5$ & $K=7$ & $K=9$ \\
set & & & & \\
\hline $1: 1$ & $90.8 \%$ & $90 \%$ & $88.3 \%$ & $85 \%$ \\
$1: 4$ & $96.7 \%$ & $95 \%$ & $91.6 \%$ & $89.2 \%$ \\
$1: 9$ & $97.5 \%$ & $95.8 \%$ & $95 \%$ & $91.6 \%$ \\
\hline
\end{tabular}

\section{5 实验算法对比及结果}

实验将 KNN 算法、SVM 算法和 CNN 算法准确 率结果进行对比, 如表 3 所示。
表 33 种算法准确率结果对比

Table 3 Comparison of the accuracy of the three algorithms

\begin{tabular}{llll}
\hline & KNN & SVM & CNN \\
\hline Walking accuracy & $100 \%$ & $94.8 \%$ & $100 \%$ \\
Sit down accuracy & $92.5 \%$ & $70.0 \%$ & $90.0 \%$ \\
Fall Accuracy & $97.5 \%$ & $82.0 \%$ & $90.0 \%$ \\
Average accuracy & $95.0 \%$ & $82.3 \%$ & $93.3 \%$ \\
\hline
\end{tabular}

由 $\mathrm{KNN}$ 算法、SVM 算法和 CNN 算法的实验分 类识别结果可知, KNN 算法识别准确率比 SVM 算法 和 CNN 算法准确率高。但 KNN 算法中坐下的识别率 较行走和跌倒低, 这是因为坐下和跌倒两种动作易造 成错误分类。

\section{5 结论}

本文提出了一种基于红外阵列传感器的人体行 为识别系统，通过提取监测区域温度分布信息的特 征，并使用 KNN 算法测得行走、坐下和跌倒的检测 准确率分别为 $100 \% 、 92.5 \%$ 和 $97.5 \% ， 3$ 种人体行为 的平均识别准确率为 $95 \%$ 。该系统在老年人生命健康 安全监护领域具有很高的应用价值。未来，可进一步 结合步态识别技术分析检测到的动作对应的人员身 份，以便在系统检测到异常行为动作时可以做出更加 准确的处理方案。

\section{参考文献:}

[1] 蔡伦, 林岑, 周䪻, 等. 老年人跌倒的公共卫生研究进展 [J]. 中国老 年学杂志, 2018, 38(9): 222-225.

CAI Lun, LIN Cen, ZHOU Zi, et al. Advances in public health research on falls of the elderly[J]. Chinese Journal of Gerontology, 2018, 38(9): 222-225.

[2] 吕汶聪, 张志静. 老年人跌倒现状及护理对策[J]. 现代医学与健康研 究电子杂志, 2018: 119-119.

LV Wencong, ZHANG Zhijing. The current situation of elderly fall and nursing countermeasures[J]. Modern Medicine and Health Research E-Magazine, 2018: 119-119.

[3] 吴志强, 曹蕾, 王凯, 等. 基于智能手机的人体跌倒检测系统设计[J]. 计算机工程与设计, 2014, 35(4): 1465-1470.

WU Zhiqiang, CAO Lei, WANG Kai, et al. Design of human body fall detection system based on smart phone[J]. Computer engineering and design, 2014, 35(4): 1465-1470.

[4] 左常玲. 基于视频的自动摔倒检测研究与实现 [D]. 合肥: 安徽大学, 2012.

ZUO Changling. Research and implementation of video-based automatic fall detection[D]. Hefei: Anhui University, 2012.

[5] 王刚. 基于多传感器的可穿戴跌倒检测系统的设计与实现[D]. 北京: 


\section{北京工业大学, 2015.}

WANG Gang. Design and implementation of wearable fall detection system based on multi-sensor[D]. Beijing: Beijing University of Technology, 2015.

[6] 李仲华, 藏春华, 杨刚. 基于噪声嵌入的跌倒检测系统的设计 [J]. 微 机处理, 2017(2): 74-76.

LI Zhonghua, ZANG Chunhua, YANG Gang. Design of fall detection system based on noise embedding[J]. Microcomputer Processing, 2017(2): 74-76.

[7] Mashiyama S, Hong J, Ohtsuki T. A fall detection system using low resolution infrared array sensor[C]// International Symposium on Personal, Indoor and Mobile Radio Communications. IEEE, 2014: 2109-2113.

[8] 杨任兵, 程文播, 钱庆, 等. 红外图像中基于多特征提取的跌倒检测 算法研究[J]. 红外技术, 2017, 39(12): 1131-1138.

YANG Renbing, CHENG Wenbo, QIAN Qing, et al. Research on fall detection algorithm based on multi-feature extraction in infrared image[J]. Infrared technology, 2017, 39(12): 1131-1138.

[9] LIU Chienliang, LEE Chiahoang, LIN Pingmin. A fall detection system using k-nearest neighbor classifier[J]. Expert System with Applications, 2010(37): 7174-7181.

[10] 张舒雅, 吴科艳, 黄炎子, 等. 基于 SVM_KNN 的老人跌倒检测算法 [J]. 计算机与现代化, 2017(12):5 3-59.

ZHANG Shuya, WU Keyan, HUANG Yanzi, et al.Old man fall detection algorithm based on SVM_KNN[J]. Computer and Modernization, 2017(12): 53-59.

[11] 陈玮, 周晴, 曹桂涛. 基于 SVM 和阈值分析法的摔倒检测系统 [J]. 计 算机应用于软件, 2017(7): 182-187.
CHEN Wei, ZHOU Qing, CAO Guitao. Fall detection system based on SVM and threshold analysis method[J]. Computer application software, 2017(7): 182-187

[12] Nadi M, Elbendary N, Hassanien A E, et al. Falling Detection System Based on Machine Learning[C]//International Conference on Advanced Information Technology \& Sensor Application. IEEE, 2016: 71-75.

[13] Adhikari K, Bouchachia H, Nait-Charif H. Activity recognition for indoor fall detection using convolutional neural network[C]//2017 Fifteenth IAPR International Conference on Machine Vision Applications (MVA). IEEE, 2017: 81-84.

[14] FENG P, YU M, Naqvi S M, et al. Deep learning for posture analysis in fall detection[C]//2014 International Conference on Digital Signal Processing (DSP). IEEE, 2014: 12-17.

[15] Vidigal M, Lima M, Neto A D A. Elder Falls Detection Based on Artificial Neural Networks[C]//Fourteenth Mexican International Conference on Artificial Intelligence. IEEE Computer Society, 2015: 226-230.

[16] Kerdegari H, Mokaram S, Samsudin K, et al. A Pervasive Neural Network Based Fall Detection System on Smart Phone[J]. Journal of Ambient Intelligence and Smart Environments, 2015, 7(2): 221-230.

[17] Linhong Wang. Human infrared signal recognition using single PIR detector[C]//2011 4th International Congress on Image and Signal Processing, 2011: 2664-2668.

[18］李洪奇, 杨中国, 朱丽萍, 等. 基于数据集特征的 $\mathrm{KNN}$ 最优 $\mathrm{K}$ 值预测 方法[J]. 计算机应用与软件, 2013, 33(6): 54-58.

LI Hongqi, YANG Zhongguo, ZHU Liping, et al. KNN optimal K-value prediction method based on data set features[J]. Computer Applications and Software, 2013, 33(6): 54-58. 